\title{
Meteoroid Risk Assessment of Lunar Habitat Concepts
}

\author{
Steven W. Evans ${ }^{1}$, Roderick Stallworth ${ }^{2}$, Jennifer Robinson ${ }^{3}$, \\ Robert Stellingwerf ${ }^{4}$, and Erich Engler ${ }^{5}$
}

${ }^{1}$ Mail Stop EM50, NASA Marshall Space Flight Center, Huntsville, AL 35812.

${ }^{2}$ Mail Stop EV23, NASA Marshall Space Flight Center, Huntsville, AL 35812.

${ }^{3}$ Jacobs-Sverdrup, Huntsville, AL 35812.

${ }^{4}$ Stellingwerf Consulting, Huntsville, AL 35803

${ }^{5}$ APL, NASA Marshall Space Flight Center, Huntsville, AL 35812

\begin{abstract}
Preliminary analyses have been performed of meteoroid impacts on lunar surface structures built with elements made from in situ materials. The SPHC hydrodynamic code was used to simulate impacts, and was validated against hypervelocity impact test results. Impact risk will affect necessary wall thicknesses, depth of burial, and placement of lunar base structures.
\end{abstract}

\section{Introduction}

Lunar habitat concept development and fabrication methods are being studied to further the manned exploration of space. The design of these structures will depend on the harsh environments they must withstand and the materials available for use. In order to achieve cost-, mass-, and volume-savings, it will be necessary to use in situ resources. Surface manufacturing and construction processes will dictate the structural elements to be used and the maturity of the overall design. In situ resources and materials will also be used to provide impact shielding for the habitat structure from meteoroids and lunar surface ejecta. It is imperative to include the effects of these impacts early in the program development to assess weight, schedule, and cost effects, and to allow integration of shielding requirements with other structural requirements.

The purpose of this study was to evaluate some design concepts and analyze the impact phenomena expected from the meteoroid and ejecta models, including simulations of high velocity impacts. Required shielding and damage to habitats placed on the lunar surface were examined, along with the risk of various failure modes due to impacts.

\section{Potential Failure Modes}

The potential failure modes caused by hypervelocity impacts are thermal and radiation protection degradation; structural degradation by partial or complete wall penetration or by internal spallation; and injury to personnel. Several analysis methods have been employed to evaluate these effects. 


\section{SPHC Impact Simulations}

The Smooth Particle Hydrodynamics impact simulation code (SPHC) was used to examine penetration and fracture of walls following impact. Code validation is an important part of this activity, and will continue as test data becomes available. Examples of some SPHC results on 1-foot-thick concrete models are shown in Figure 1 , and damage estimation curves developed from these simulations are shown in Figure 2.

\section{Meteoroid Impact on a 1 Foot Thick Concrete Slab}

Impactor $=$ sphere at $\mathrm{V}=20 \mathrm{~km} / \mathrm{s}$, density $=0.5$, stony.

Blue means damaged material.

Particle Radius $=0.25 \mathrm{~cm}$

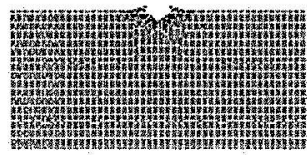

$1.0 \mathrm{~cm}$

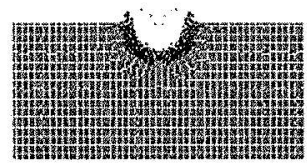

$0.50 \mathrm{~cm}$

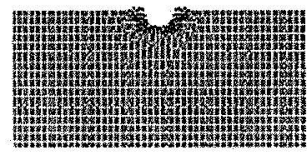

$2.0 \mathrm{~cm}$

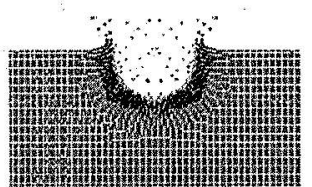

Figure 1. Examples of SPHC concrete impacts.

\section{Meteoroid Impact on a 1 Foot Thick Concrete Slab}

Impactor $=$ sphere at $\mathrm{V}=20 \mathrm{~km} / \mathrm{s}$, density $=0.5$, stony.

Particle Radius $=4.0 \mathrm{~cm}$

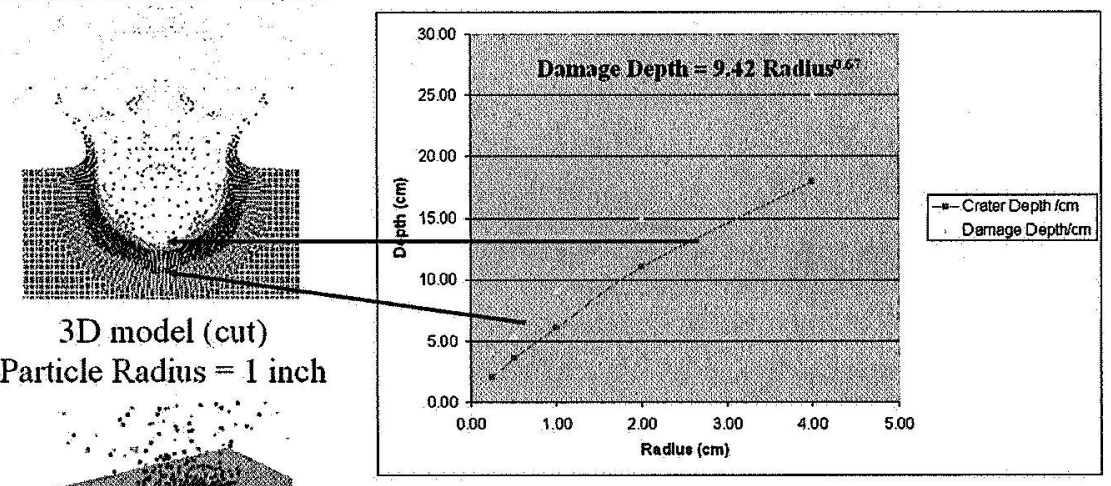

These results can be used to estimate the damage from any impact on a specific habitat candidate.

Figure 2. Sample damage estimation curves. 
A hemispherical concrete-walled dome structure having a diameter of 30 feet and a wall thickness of 2 feet is shown in Figure 3, as modeled by SPHC. A 1-bar atmosphere is modeled to show effects such as pressure loss and pressure pulse after wall perforation. The moon's gravity is also modeled. The impactor is a $20 \mathrm{~cm}$ diameter, $2.1 \mathrm{~kg}$, porous stoney meteoroid, striking at $20 \mathrm{~km} / \mathrm{sec}$.
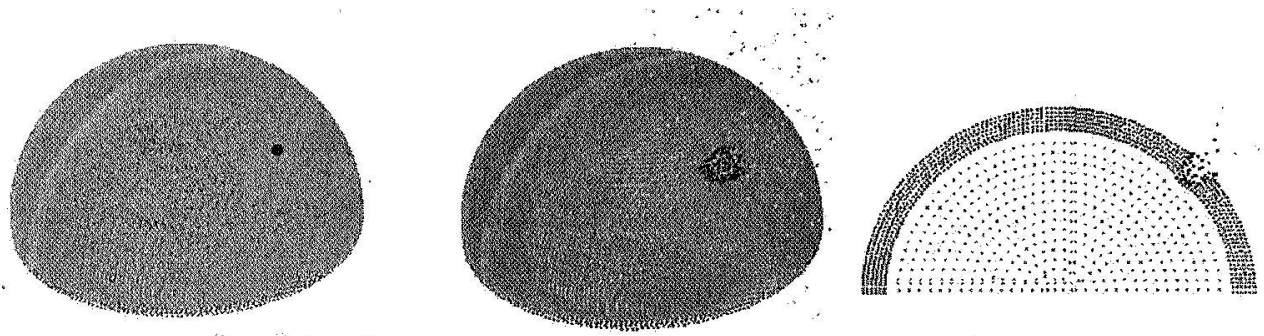

Figure 3. Dome structure impact simulation.

The left figure shows the particle just before wall impact. The middle and right figures show the impact damage and depth of penetration into the wall during the impact process. The dark blue fractured material is surrounded by a light blue plastic wave traveling around the hemispherical dome. Although this model does not indicate immediate wall perforation, late time effects are likely to cause wall failure.

\section{Example Risk Assessment}

Estimated critical particle sizes from SPHC and from traditional penetration equations were combined with existing meteoroid and surface ejecta environment models to determine the risk of failures from various modes for the baseline mission parameters.

The meteoroid and lunar surface ejecta environments were obtained from NASA SP-8013. Using this data, a set of curves for various mission lifetimes was generated from standard penetration equations for aluminum. These preliminary curves were used to formulate the analytical methodology. Figure 4 shows the curves for meteoroid assessments.

Historically, initial design requirements for NASA missions have been set at a 95\% Probability of No Critical Failure (PNCF) for impacts. A shield design concept for the dome area could be determined as required by this number, to prevent critical damage to possible habitat elements such as windows or antennae.

\section{Alternative Habitat Concept Assessment}

An alternative lunar habitat consisting of a cylindrical structure 30 feet high, with a diameter of 26 feet, is shown in Figure 5 (Meyers, 2005). The exterior area of such a structure that is exposed to meteors is about $330 \mathrm{~m}^{2}$. The proposed wall thickness is 6 inches of processed regolith. The survivability of such a structure with 
respect to particle impact was estimated using an analytical approach consisting of the above meteoroid flux model, lunar ejecta flux model, and penetration results derived from a series of SPHC models of particle impacts on concrete blocks.

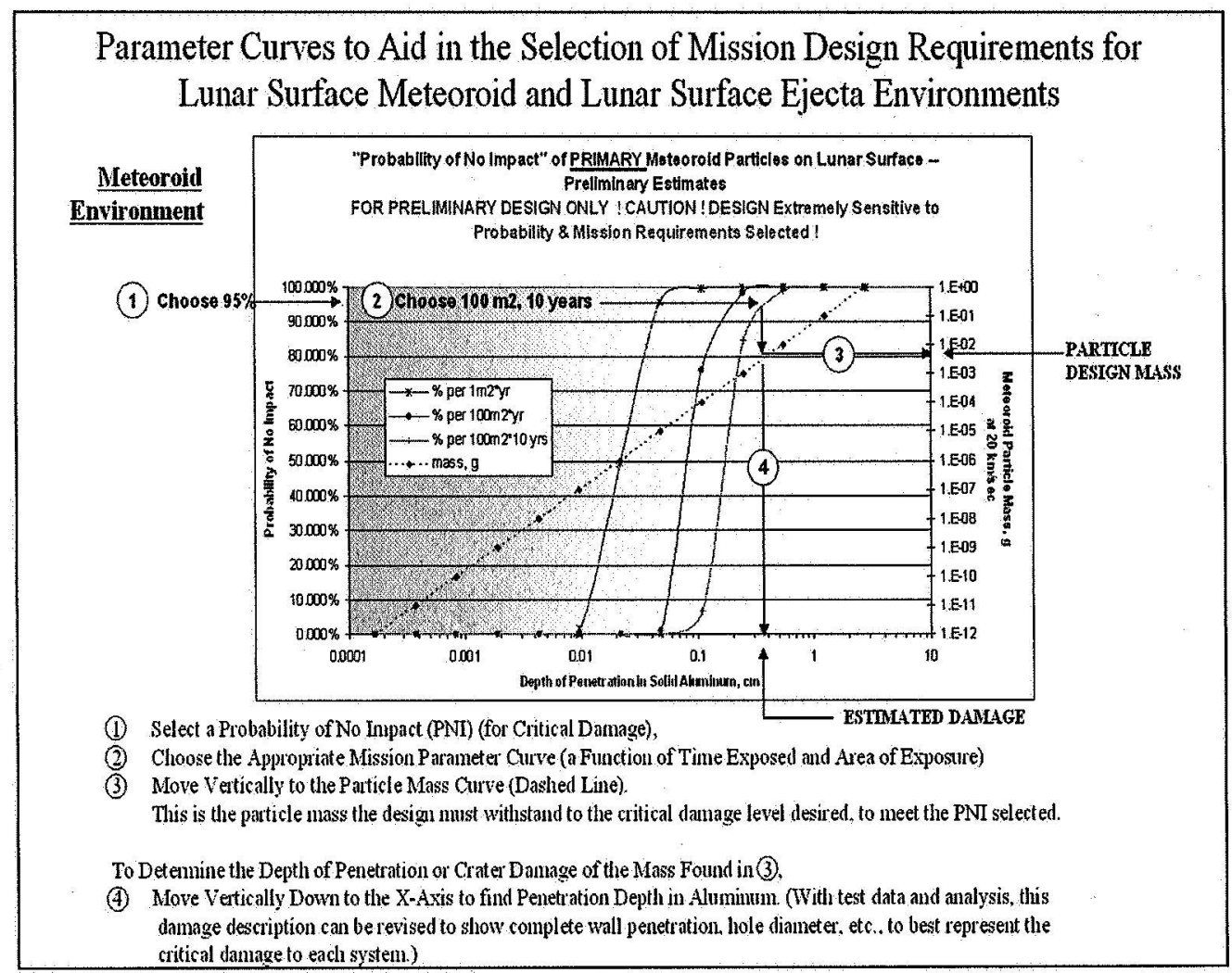

Figure 4. Design curve for meteoroid assessments.

Several models in the SPHC sequence used to examine damage to a six-inch concrete wall are shown in Figure 6. All of the models simulated meteoroid impacts with velocity $20 \mathrm{~km} / \mathrm{s}$ and density $0.5 \mathrm{~g} / \mathrm{cc}$ (stony meteoroids). The radii of the particles used are shown in the figure. The crater depths of the models are well represented $\left(\mathrm{R}^{2}=0.990\right)$ by the following fit to the data:

$$
D=4.34 * d
$$

where $D$ is the crater depth in $\mathrm{cm}$ and $d$ is the particle diameter in $\mathrm{cm}$.

In order to include the ejecta damage (which occurs at a much lower velocity), additional models were run at velocities of $100 \mathrm{~m} / \mathrm{s}$ to $20 \mathrm{~km} / \mathrm{s}$, for several densities of particle. The following fit represents the full data set with an $\mathrm{R}^{2}=0.997$ :

$$
D=1.545 * \rho^{0.33} * V^{0.67} * r
$$

where $D$ is crater depth in $\mathrm{cm}, \rho$ is projectile density in $\mathrm{g} / \mathrm{cm}^{3}, V$ is projectile velocity in $\mathrm{km} / \mathrm{s}$, and $r$ is projectile radius in $\mathrm{cm}$. 


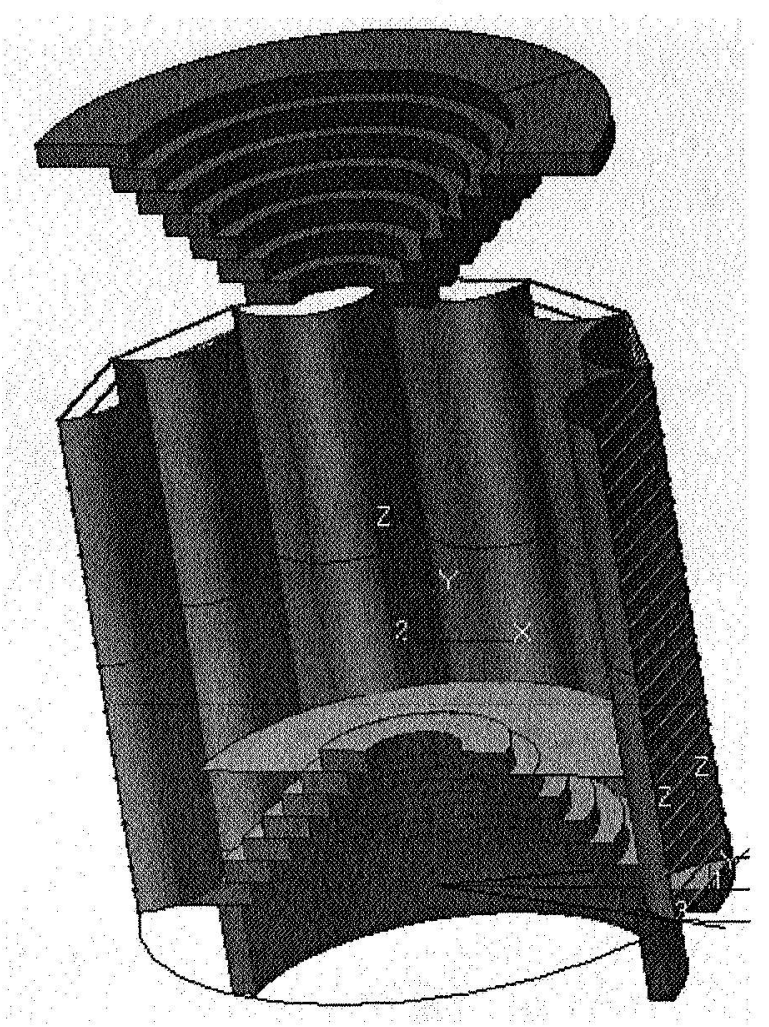

Figure 5. Alternative lunar habitat concept.
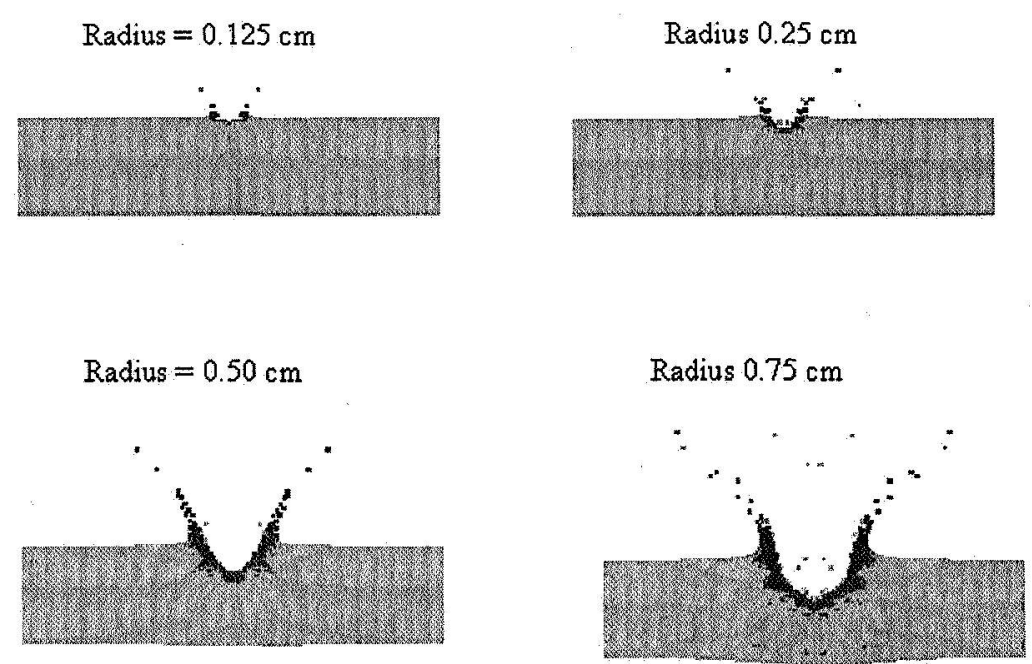

Figure 6. Four SPHC models in the sequence of 6 -inch-thick concrete walls.

Figure 7 shows the Probability of No Penetration (PNP) for a concrete lunar structure with an external area of $330 \mathrm{~m}^{2}$, for a period of 20 years. The X-axis is the wall thickness; the left curve shows the ejecta result, while the right curve shows the 
meteoroid result. This is considered to be a conservative estimate of the survivability of the structure, considering solid particle impacts. The concrete wall is considered "penetrated" when the penetration depth exceeds $1 / 3$ of the total thickness of the wall. At this point the concrete wall is likely to be severely damaged, with secondary damage (cracking) possibly extending through the entire wall. The PNP for ejecta is 0.9999 , while that due to meteoroids is 0.99 , giving an overall PNP of 0.99 . For meteoroids the critical case has a mass of $0.15 \mathrm{~g}$ and a diameter of $0.83 \mathrm{~cm}$. For the ejecta the critical case has a mass of $6 \mathrm{~kg}$ and a diameter of $16.8 \mathrm{~cm}$. These differences are primarily due to the differing impact speeds.

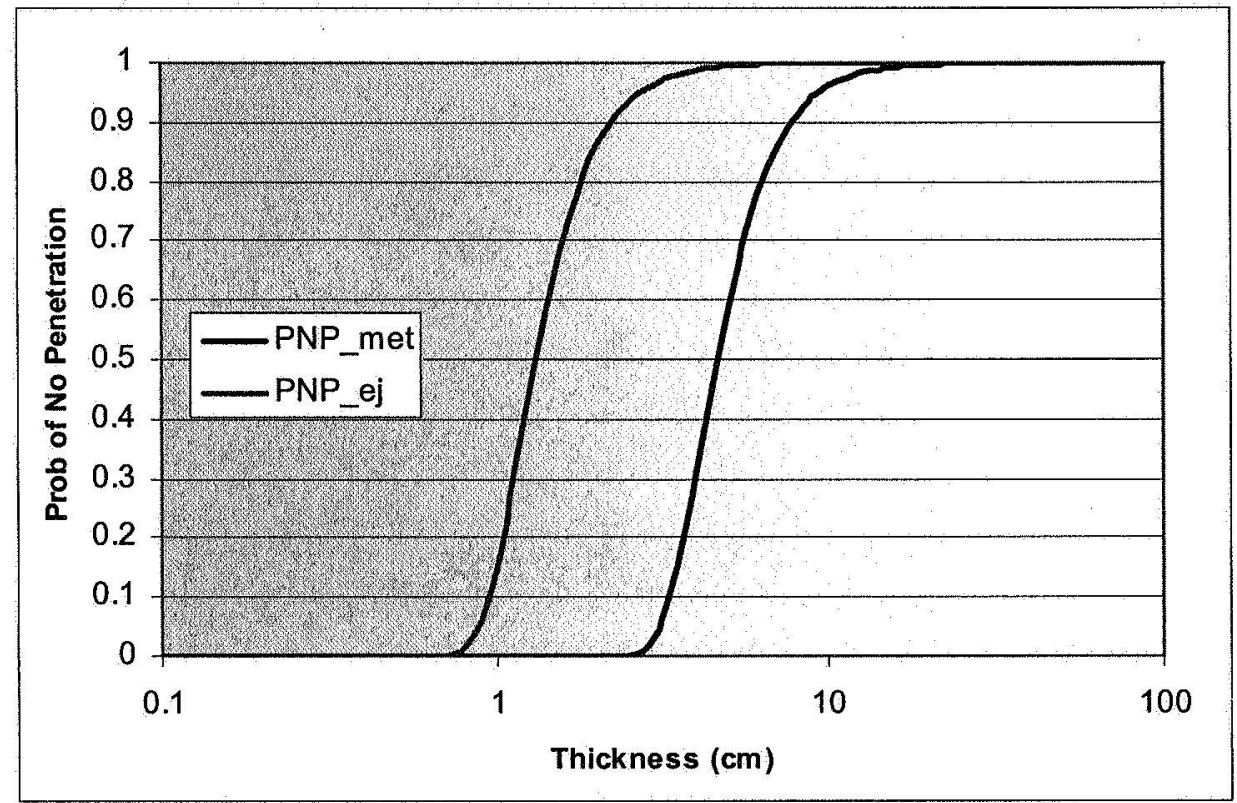

Figure 7. Probability of No Penetration (PNP) for meteoroids (right curve) and surface ejecta (left) versus wall thickness for concrete structures.

\section{Hypervelocity Impact Test Results}

A hypervelocity impact test was conducted, having as its target a $5 \times 5 \times 5-\mathrm{cm}$ sample of sulfur concrete. This item consisted of JSC-1 Lunar Regolith Simulant $(65 \%)$ and sulfur $(35 \%)$. The sample was placed in the MSFC Impact Test Facility's Micro Light Gas Gun' target chamber, and was struck by a 1-mm diameter $(\sim 1.4 \mathrm{e}-03$ g) aluminum projectile at $5.85 \mathrm{~km} / \mathrm{s}$. Figure 8 shows a photo of the sample after the impact. A rather conical crater was produced in the target face measuring $12.8 \mathrm{~mm}$ in average diameter $(6.4 \mathrm{~mm}$ radius) and $3.1 \mathrm{~mm}$ in central depth. Surrounding the crater is a zone of fractured material, which appears as a crenate cracked area $\sim 8-12$ $\mathrm{mm}$ from the crater center, covering $\sim 140$ degrees, and exhibiting an attached flake and two scars from detached flakes, each $\sim 3-4 \mathrm{~mm}$ long and $2 \mathrm{~mm}$ wide.

Figure 9 shows the results of a simulation of this impact. The simulation crater is $\sim 15 \mathrm{~mm}$ in diameter and $3.5 \mathrm{~mm}$ deep - in good agreement with the test crater 
dimensions - and shows a similar conical profile. Note the fractures (dark blue) extending about $1 \mathrm{~cm}$ into the sample below the crater. Grid lines are every $0.4 \mathrm{~cm}$.

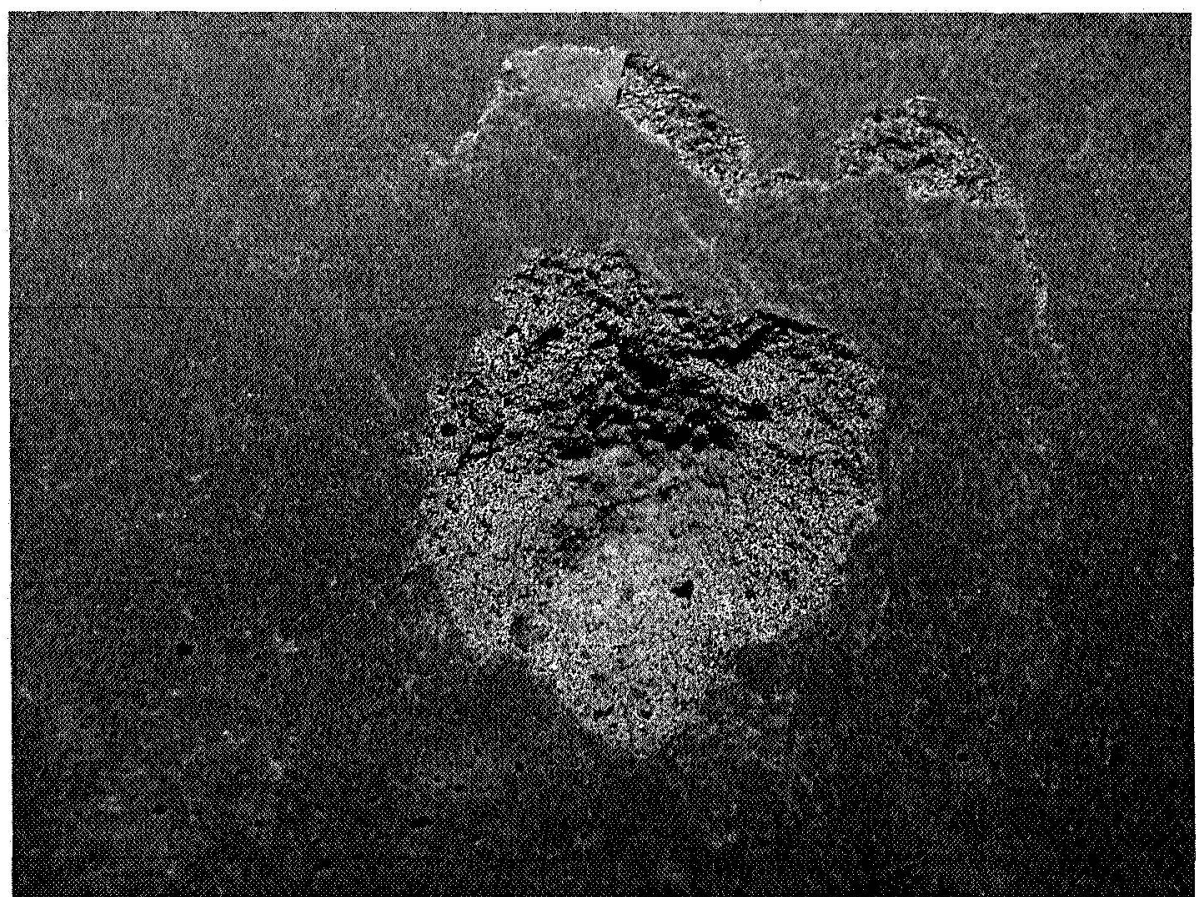

Figure 8. Sulfur concrete sample after impact by 1-mm diameter aluminum projectile at $5.85 \mathrm{~km} / \mathrm{s}$.

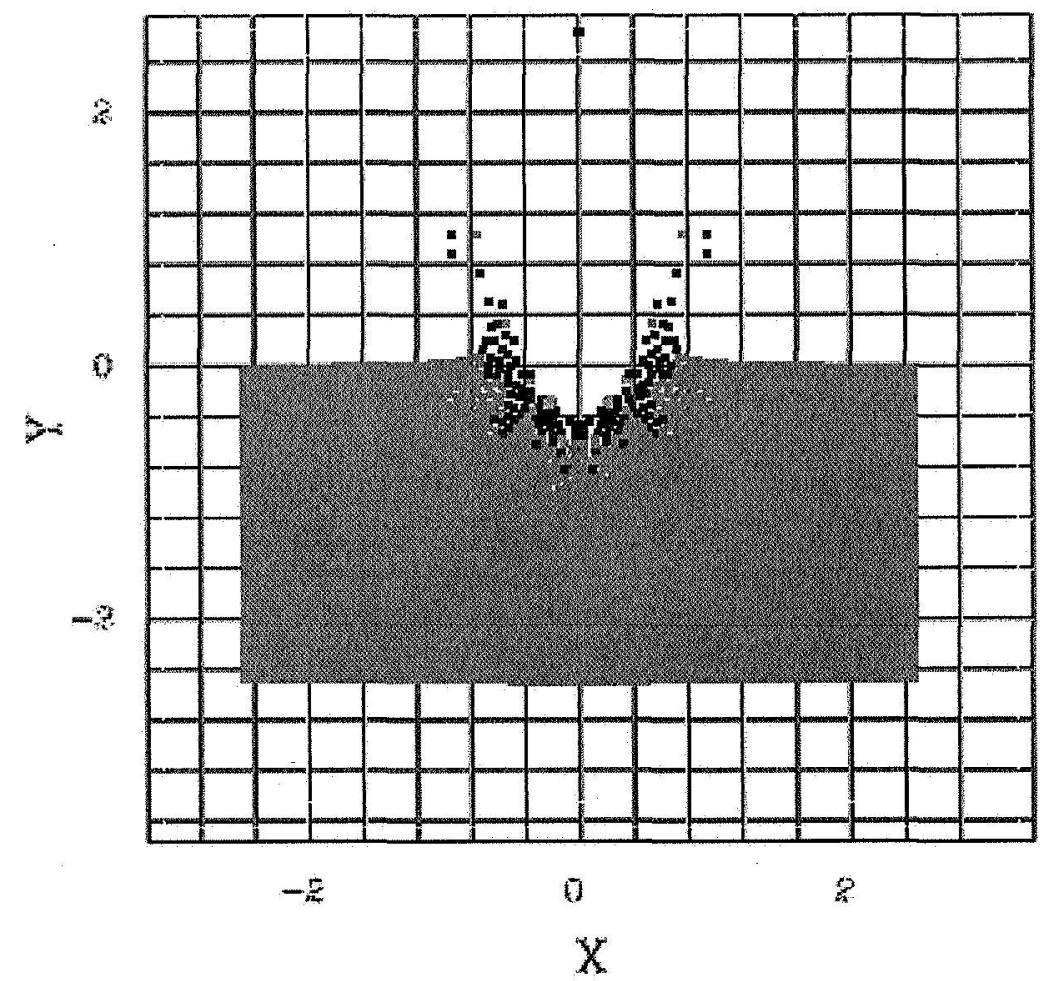

Figure 9. Cross section of an SPHC model of the experimental shot. 


\section{Conclusions}

For the 30-foot-tall silo structure considered here, the probability of serious impact damage over a 20 year interval is found to be about $1 \%$. If ten of these structures were built to form a lunar base, the probability of wall penetration of one of the structures would increase to about $10 \%$, an unacceptable level for manned structures. These are preliminary results, and a more detailed analysis will be needed for each candidate design. This result does clearly show, however, the importance of a meteoroid threat analysis for any fixed surface base on the moon, and the need to design the structures with this threat in mind. In particular, the identification of important or vulnerable areas on the structure could strongly affect the final result.

Most meteor impacts with be from micrometeoroids. Although not a direct threat to the walls of a structure, vital communications equipment, external sensors, solar power generators, thermal radiators, etc., will be more sensitive to small impacts. Over time, erosion of tougher structures will occur. Impact models can be used to evaluate these types of effects.

Another consideration is the effect of a large meteor impact on or near the lunar base. Large impacts do occur every day on the moon, and even though the probability of a nearby strike is very small, it could happen. Some warning might be available if a large impactor is dangerously near; in any case, a plan for evacuation to shelter, erection of a temporary shield, or relocation of the crew to a safer location should be developed in case of a catastrophic impact. A detailed analysis of the damage caused by such an event could help constrain the size, shape, and placement of individual structures in the base to minimize detrimental effects.

Only a single experimental test has been done on simulated regolith material. Although the models of impacts on concrete agree reasonably well with this test, more data on a variety of lunar construction materials are needed. Once fully validated on several candidate materials, hydrodynamic models can predict damage at realistic velocities not attainable by experiments $(20 \mathrm{~km} / \mathrm{s}$, vs. experiments at $7 \mathrm{~km} / \mathrm{s})$. The models can also be used in the design stage to evaluate the relative survivability of different structures.

\section{References}

Meyers, C., internal structural design memorandum to Marshall Space Flight Center In-situ Resource Utilization Project Office, Summer 2005.

NASA SP-8013, "Meteoroid Environment Model: Near Earth to Lunar Surface," 1969. 